\title{
Single-Ended Oscillation-Based Test Technique for Passive RF Phase Shifters
}

\author{
Johannes J.P. Venter ${ }^{*}$, Tinus Stander ${ }^{+}$, \\ Carl and Emily Fuchs Institute for Microelectronics, Dept. EEC Engineering, \\ University of Pretoria, South Africa \\ *Venter.JJP@tuks.co.za, ${ }^{+}$Tinus.Stander@up.ac.za
}

\begin{abstract}
Phased array systems have become paramount to the development of next generation wireless communication networks, with errors on phase shifters contributing to beam pointing error. Oscillation-based built-in self-testing (OBIST) of phase shifters may improve on this accuracy. We propose a singleended oscillation-based test technique for passive RF phase shifters. The method uses a negative resistance oscillator topology, where the phase shifter is used to capacitively load the active negative resistance circuit resulting in a dependency between relative phase shift and output oscillation frequency. Simulation results indicate an average sensitivity of $0.14 \mathrm{MHz} /{ }^{\circ}$ of phase shift around a nominal $617 \mathrm{MHz}$ output oscillation when testing an Xband reflection-type phase shifter, while the addition of OBT circuitry increases the phase shifter's mid-band insertion loss by $0.93 \mathrm{~dB}$.
\end{abstract}

Keywords - Phase shifters, oscillation-based test, built-in selftest, phased arrays.

\section{INTRODUCTION}

Phased array systems have become an integral part of next generation communication systems, due to the flexibility of dynamic beam steering to optimize data speeds and minimize interference [1]. In analog or hybrid beamforming [2], phase shifters are required to effect beam steering. Phase shifters have imperfect phase tuning performance, resulting in an error in beam pointing angle [3]; a reduction in relative phase shift error would, therefore, aid the improvement of overall system performance [3], [4]. It has been proposed that oscillation-based test (OBT) methods for phase shifters can be utilized to improve phased array system performance [3], [5], as a calibrated RF source is not required to generate an input test vector.

OBT techniques have been applied to various RF circuits such as analogue-to-digital converters, active and passive filters, low-noise amplifiers, as well as mm-wave phase shifters [5], [6]. The technique in [5] transforms the phase shifter, or device under test (DUT), into an oscillator using a cross-coupled oscillator topology. This method uses quarter wavelength transmission lines to isolate the OBT circuitry from the phase shifter, requiring no series switches. While this is advantageous, given the loss and variability of the required switches, the isolation circuitry would be too large to implement on-chip for lower frequencies.

In [6], embedded passive RF transmission lines and filters are tested using a single-ended OBT method by loading an existing RF oscillator with the DUT and monitoring the variation in oscillation frequency. In this work, we extend on this approach to present a single-ended OBT technique with which discrete passive RF phase shifters can be tested, which paves the way for oscillation-based built-in self-test (OBIST) implementations of phased array front-ends. Using a singleended measurement technique minimizes the number of switches in the signal path, resulting in reduced losses incurred due to the OBT circuitry.

\section{PROPOSED APPROACH}

Fig. 1 shows the canonical form of a negative resistance RF oscillator circuit [6]. For oscillation to occur the following condition needs to be satisfied [6]:

$$
Z_{I N}(\omega)+Z_{L}(\omega)=0
$$

where $Z_{\mathrm{IN}}(\omega)=R_{\mathrm{IN}}+j X_{\mathrm{IN}}$ is the complex input impedance of the negative resistance device and $Z_{\mathrm{L}}(\omega)$ is the load (resonator) impedance.

For negative resistance oscillators using negative conductance circuits, a commonly used rule-of-thumb states that [7]

$$
R_{L}=\frac{-R_{I N}}{3}
$$

where $R_{\mathrm{L}}$ and $R_{\mathrm{IN}}$ are the real parts of the small-signal load resistance and negative resistance, respectively. With the condition in (2) being met, the main determinant of the oscillation frequency is where the load and negative conductance circuit reactance values cancel out [7]:

$$
X_{L}=-X_{I N}
$$

Assuming that the real part of the load impedance $\left(R_{\mathrm{L}}\right)$ remains constant across frequency, it can be shown that when $X_{\mathrm{IN}}$ is varied the condition in (3) will be satisfied at a different frequency point. This creates a dependency between the $X_{\mathrm{IN}}$ value of the negative resistance and the oscillation frequency of the resulting oscillator. Given this, it is possible to create a frequency controllable oscillator, when the reactance part of the negative resistance circuit is made tunable, as shown in Fig. 2. This concept may be applied to OBT of passive RF phase shifters.

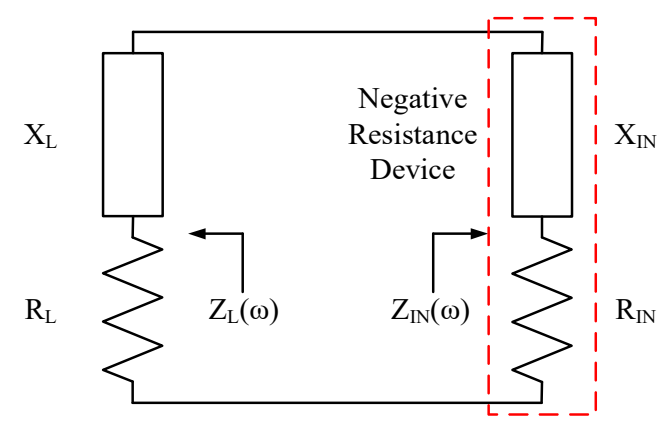

Fig. 1. Canonical RF circuit for negative resistance oscillator 


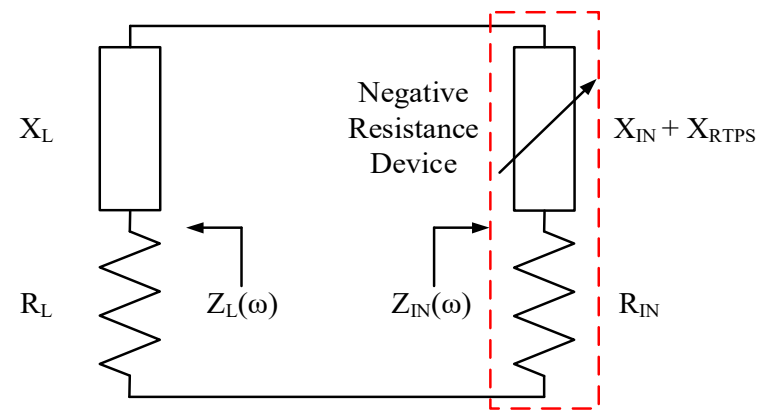

Fig. 2. Conceptual representation of proposed OBT method

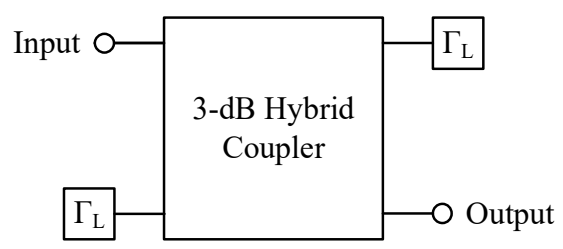

Fig. 3. Architecture diagram of RTPS

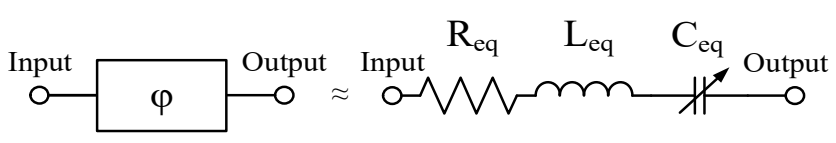

Fig 4. RLC equivalent circuit of RTPS

A common passive phase shifter topology is a reflectivetype phase shifter (RTPS). The RTPS consists of a 3-dB hybrid coupler and reflective loads placed at the through- and coupledports [5], [8]. A block diagram of an RTPS is shown in Fig. 3, where $\Gamma_{\mathrm{L}}$ represents the reflective loads.

A RTPS at frequencies outside the passband of the hybrid coupler may be modelled, approximately, as a series RLC circuit, as illustrated in Fig. 4. The equivalent capacitance of the circuit $\left(C_{e q}\right)$ varies as the RTPS relative phase shift is varied by changing the biasing of the tuning varactors; the values for $L_{e q}$ and $R_{e q}$ remain relatively constant. When considering the phase shifter outside the passband, the value of input impedance would be extremely large replicating an open circuit. This enables the RTPS to be used as capacitive loading, below the resonance of the equivalent RLC circuit, in the active negative conductance circuitry. Thus, variance in $C_{e q}$ (Fig. 4) results in a change in $X_{\text {RTPS }}$ (Fig. 2) which changes the frequency point at which the condition in (3) is satisfied. This creates a dependence between the oscillation frequency of the resultant OBT circuit and the relative phase shift of the RTPS. Such an OBT circuit (conceptually represented in Fig. 2) may be used to test and calibrate RTPS relative phase shift.

\section{IMPLEMENTATION}

A commonly used negative conductance circuit using one transistor and two positive feedback capacitors is shown in Fig. 5 [7]. For a negative conductance circuits, the best load (resonator) topology to use is a series topology [7] also shown in Fig. 5.

The DUT (phase shifter under test) is incorporated into the active negative resistance circuitry prior to designing the RF oscillator. The oscillation frequency range of the RF oscillator should be chosen in such a way that maximum variation in reactance is achieved across the RTPS phase shift range.

The design methodology employed in [8] was used to implement the RTPS, as this architecture is simple and achieves wideband operation which is of particular importance in practical phased array systems. A detailed circuit diagram of the reflective loads used in the RTPS, along with the modelling of the varactor parasitics, are shown in Fig. 6 .

The transistor used in the negative conductance circuit is the Infineon BFP740F NPN RF heterojunction bipolar transistor (HBT), and for the single pole double throw (SPDT) switch at the output of the RTPS a MACOM MASW-002103-1363 PIN diode switch is used due to its low insertion loss at X-band. The varactors used for the RTPS design are the same varactors (MACOM MA46580) used in [8], with a total capacitance ranging from $0.13 \mathrm{pF}$ to $2.2 \mathrm{pF}$, which has a parasitic series inductance of $L_{S}=0.27 \mathrm{nH}$, a series resistance of $R_{S}=2.2 \Omega$, and a parallel capacitance of $C_{P}=0.108 \mathrm{pF}$. Table I summarizes the biasing conditions and component values of the OBT circuit.

The oscillation output node is across the collector resistance (indicated by $\mathrm{V}_{\text {mon }}$ in Fig. 5) of the negative conductance circuit. This node is well isolated from the rest of the circuitry, resulting in negligible change in oscillation frequency for various port impedance values. This facilitates easy access to the OBT circuit output to measure the oscillation frequency. It is also important to note that the impedance seen at the input of the RTPS has a negligible effect on the performance of the OBT circuit. This is because the oscillation frequency of the OBT circuit is in the stopband of the hybrid coupler used in the RTPS, with $C_{e q}$ forming an effective high impedance between the test circuitry and the input of the phase shifter.

Fig. 7 conceptually illustrates how the proposed OBT method may be used, in an OBIST system, to calibrate and test RF phase shifters. The phase shifter is switched from normal operation in the RF front-end chain into OBT mode using the SPDT switch. In OBT mode there is a signal processing block that monitors, down-converts, and digitizes the RF oscillation frequency. Thereafter, there is a control block that processes the digital data and computes the changes that need to be made in the control signals of the RF phase shifter to optimize receiver performance.

TABLE I

SUMMARY OF OBT CIRCUIT PARAMETERS

\begin{tabular}{cc}
\hline \hline Parameter & Value \\
\hline $\mathrm{V}_{\mathrm{C}}$ & $3 \mathrm{~V}$ \\
$\mathrm{~V}_{\mathrm{B}}$ & $1 \mathrm{~V}$ \\
$\mathrm{I}_{\mathrm{E}}$ & $3.3 \mathrm{~mA}$ \\
\hline $\mathrm{L}_{\mathrm{L}}$ & $13.0 \mathrm{nH}$ \\
$\mathrm{C}_{\mathrm{L}}$ & $15.8 \mathrm{pF}$ \\
$\mathrm{R}_{\mathrm{L}}$ & $10.4 \Omega$ \\
$\mathrm{C}_{1}$ & $12.8 \mathrm{pF}$ \\
$\mathrm{C}_{2}$ & $13.5 \mathrm{pF}$ \\
$\mathrm{R}_{\mathrm{OSC}}$ & $50 \Omega$ \\
\hline \hline
\end{tabular}




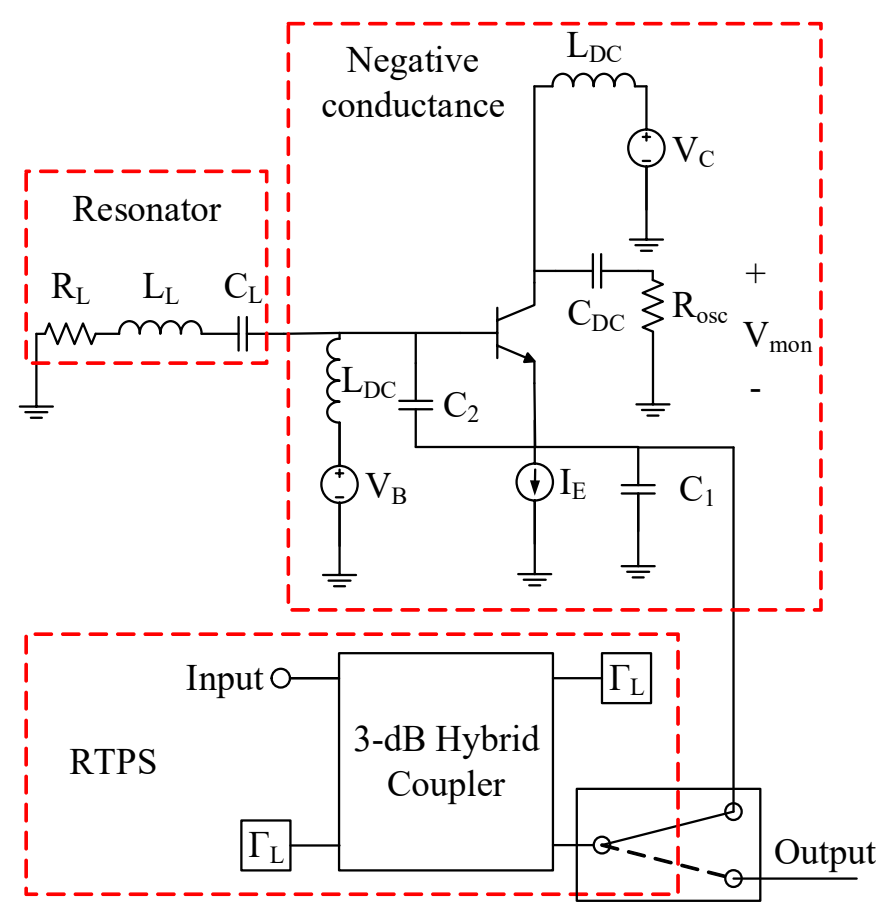

Fig. 5. Schematic of proposed OBT circuit, with circuit in OBT mode

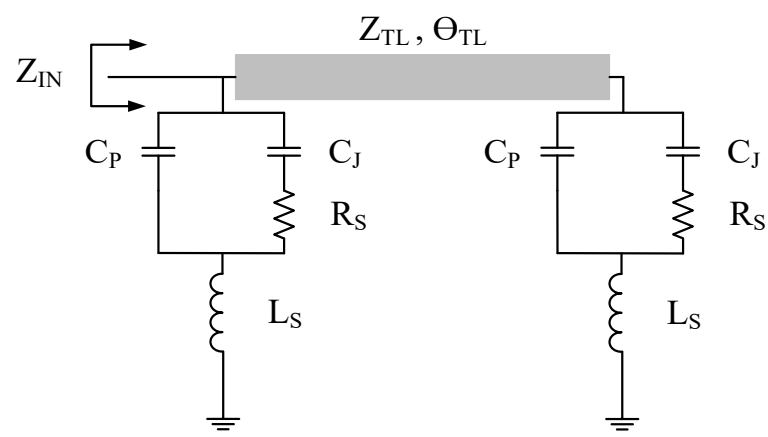

Fig. 6. Schematic of RTPS reflective loads ( $\Gamma_{\mathrm{L}}$ in Fig. 5)

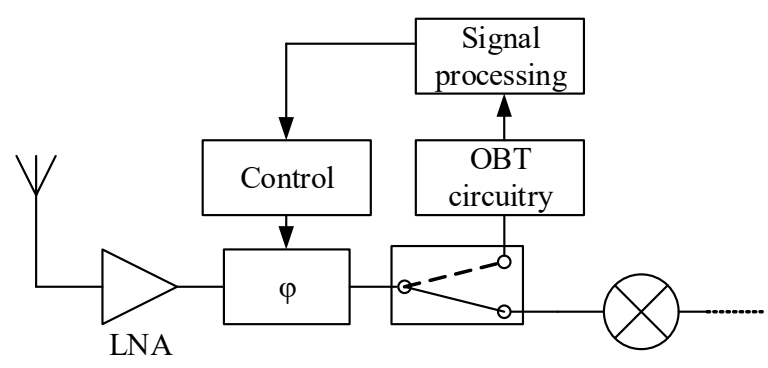

Fig. 7. High level representation of phase shifter OBIST concept in RF receiver chain

\section{SimUlation RESUltS}

All simulations were conducted in Keysight ADS 2018 using the harmonic balance solver for the OBT circuit and the linear circuit solver for the determination of the S-parameter results. Simulations used ideal transmission line components, with transistor, SPDT switch, and varactor models included, as supplied by the respective manufacturers.
The OBT oscillation frequency $f_{0}$ and the RTPS relative phase shift for various varactor bias voltages is shown in Fig. 8. The RTPS exhibits $373^{\circ}$ phase shift range at $10 \mathrm{GHz}$ resulting in an average sensitivity of $0.14 \mathrm{MHz} /{ }^{\circ}$. The OBT circuit is much more sensitive to change in RTPS relative phase shift values below $150^{\circ}$, with minimal change in oscillation frequency for larger relative phase shifts. This is a drawback of the current solution and would require further investigation to increase the sensitivity for larger relative phase shifts. Fig. 9 shows the oscillation output magnitude varies from $-0.77 \mathrm{dBm}$ to $-2.7 \mathrm{dBm}$.

The average and worst-case S-parameter results of the RTPS, with and without the OBT circuitry, is shown in Fig. 10. The transmission response $\left(\mathrm{S}_{21}\right)$ shape remains similar with the addition of $0.93 \mathrm{~dB}$ insertion loss, at $10 \mathrm{GHz}$, for the RTPS with OBT due to the added series losses of the SPDT switch placed at the output of the RTPS. There is minimal additional return loss at the lower end of the RTPS passband, however, the return loss increases for the upper passband frequencies. Minor reduction $(880 \mathrm{MHz})$ in the $-10 \mathrm{~dB}$ input match passband is observed.

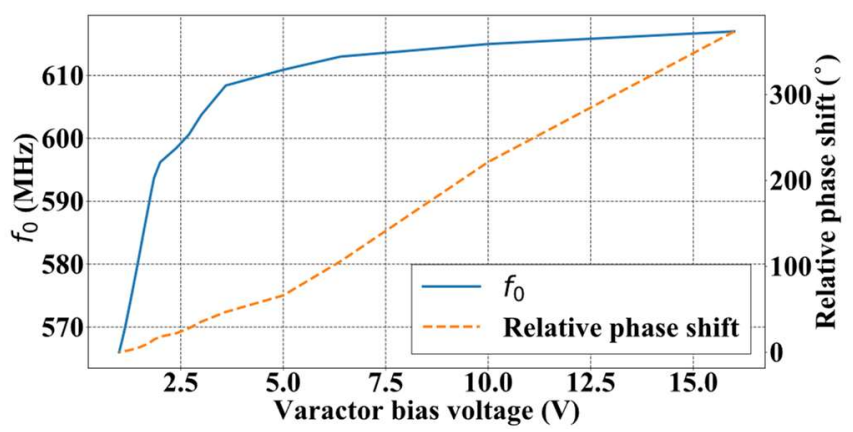

Fig. 8. OBT $f_{0}$ and RTPS relative phase shift for various varactor bias voltages

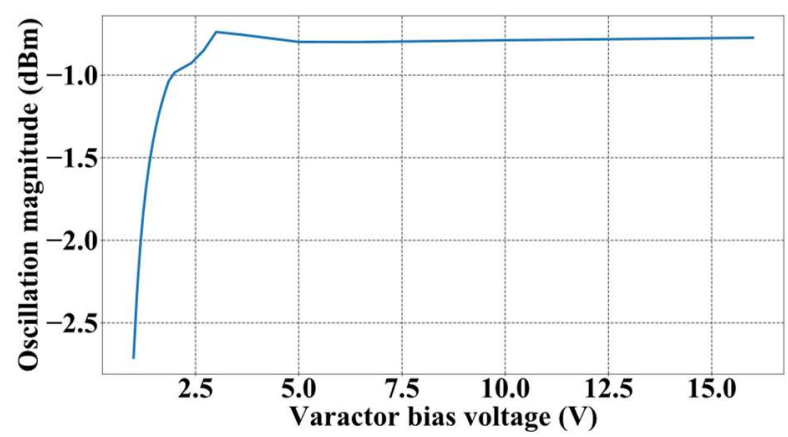

Fig. 9. Oscillation magnitude for various varactor bias voltages 


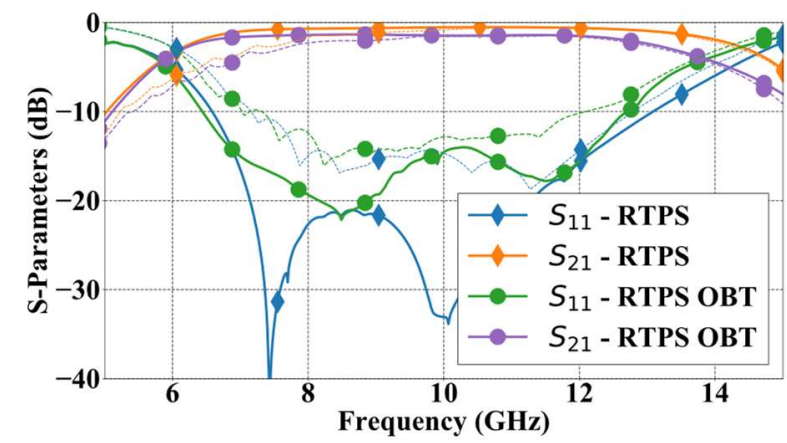

Fig. 10. Comparison of average RTPS S-parameter performance over tuning capacitance range with and without OBT circuitry (Worst case responses indicated in dashed line)

\section{CONCLUSION}

We have presented a single-ended oscillation-based test technique for the characterization of passive RF phase shifters. The OBT technique consists of a negative resistance oscillator topology, where the active negative resistance is loaded with the phase shifter under test. This configuration results in a variation of the reactance of the active negative resistance for various phase shifter phase states, which changes the oscillation frequency of the OBT circuit. The method makes use of a single port, which reduces the degradation in phase shifter performance when the OBT circuitry is added.

A proof-of-concept design is presented in simulation carried out using a RTPS phase shifter. It was observed that the circuit exhibits an average sensitivity of $0.14 \mathrm{MHz} /{ }^{\circ}$ to relative phase shift over a relative phase shift range of $373^{\circ}$. There is marginal degradation in the phase shifter S-parameter performance $(0.9$ $\mathrm{dB}$ additional insertion loss and $880 \mathrm{MHz}$ reduction in $-10 \mathrm{~dB}$ input match bandwidth) due to the OBT circuitry.

Future work will include improving sensitivity for large relative phase shift states, prototyping and characterizing the detectability of phase shift error under stress, as well as implementing differential power detection of $f_{0}$ as demonstrated in [9], [10].

\section{ACKNOWLEDGMENT}

Acknowledgements redacted for double-blind review.

\section{REFERENCES}

[1] S. Jeon, Y.-J. Wang, H. Wang, F. Bohn, A. Natarajan, A. Babakhani and A. Hajimiri, "A Scalable 6-to-18 GHz Concurrent Dual-Band QuadBeam Phased-Array Receiver in CMOS," IEEE Journal of Solid-State Circuits, vol. 43, no. 12, pp. 2660-2673, Dec. 2008.

[2] I. Ahmed, H. Khammari, A. Shahid, A. Musa, K. S. Kim, E. De Poorter and I. Moerman, "A Survey on Hybrid Beamforming Techniques in 5G: Architecture and System Model Perspectives," IEEE Communications Surveys \& Tutorials, vol. 20, no. 4, pp. 3060-3097, 2018.

[3] J. W. Jeong, J. Kitchen and S. Ozev, "A Self-Compensating Built-In SelfTest Solution for RF Phased Array Mismatch," in 2015 IEEE International Test Conference (ITC), Anaheim, CA, 2015.

[4] M. Shafiee and S. Ozev, "Contact-Less Near-Field Measurement of RF Phased Array Antenna Mismatches," in 2017 22nd IEEE European Test Symposium (ETS), Limassol, Cyprus, 2017.

[5] M. Margalef-Rovira, M. J. Barragan, E. Sharma, P. Ferrari, E. Pistono and S. Bourdel, "An Oscillation-Based Test Technique for On-Chip Testing of mm-Wave Phase Shifters," in 2018 IEEE 36th VLSI Test Symposium (VTS), San Francisco, CA, 2018.

[6] A. Goyal, M. Swaminathan and A. Chatterjee, "One-Port ResonanceBased Test Technique for RF Interconnect and Filters Embedded in RF Substrates," IEEE Transactions on Components, Packaging and Manufacturing Technology, vol. 3, no. 2, pp. 236-246, Feb. 2013.

[7] R. Ludwig and G. Bogdanov, "Chapter 10.1.2: Negative Resistance Oscillators," in RF Circuit Design: Theory and Applications, New Jersey, Pearson Education, Inc., 2009, pp. 562-578.

[8] J. J. P. Venter and T. Stander, "X-band Reflection-Type Phase Shifters Using Coupled Line Couplers on Single Layer RF PCB," IEEE Microwave and Wireless Components Letters, vol. 28, no. 9, pp. 807809, Sep. 2019.

[9] M. Ballot and T. Stander, "Built-in Oscillation-Based Testing of RF Amplifier Gain using Differential Power Detection," in 2019 IEEE Radio and Antenna Days of the Indian Ocean (RADIO), Reunion, 2019.

[10] H. P. Nel, T. Stander and F. C. Dualibe, "Built-In Oscillation-Based SelfTesting of a $2.4 \mathrm{GHz}$ LNA in 0.35 um CMOS," in 2018 25th IEEE International Conference on Electronics, Circuits and Systems (ICECS), Bordeaux, 2018. 